\title{
Genetic contributions to age-related decline in executive function: a 10-year longitudinal study of COMT and BDNF polymorphisms
}

\author{
Kirk I. Erickson ${ }^{1,2,3, *}$, Jennifer S. Kim ${ }^{2,3}$, Barbara L. Suever ${ }^{4}$, Michelle W. Voss ${ }^{1,3}$, \\ B. Magnus Francis ${ }^{5}$ and Arthur F. Kramer ${ }^{1,2,3}$ \\ 1. Psychology Department, University of Illinois at Urbana-Champaign, Champaign, IL, USA \\ 2. Neuroscience Program, University of Illinois at Urbana-Champaign, Urbana, IL, USA \\ 3. Beckman Institute for Advanced Science and Technology, University of Illinois at Urbana-Champaign, Urbana, IL, USA \\ 4. School of Integrative Biology, University of Illinois at Urbana-Champaign, Urbana, IL, USA \\ 5. Entomology Department, University of Illinois at Urbana-Champaign, Urbana, IL, USA
}

Edited by: $\quad$ William J. Jagust, University of California Berkeley, USA

Reviewed by: Cheryl Grady, University of Toronto, Canada

Roshan Cools, University of Cambridge, UK

\begin{abstract}
Genetic variability in the dopaminergic and neurotrophic systems could contribute to age-related impairments in executive control and memory function. In this study we examined whether genetic polymorphisms for catechol-O-methyltransferase (COMT) and brainderived neurotrophic factor (BDNF) were related to the trajectory of cognitive decline occurring over a 10-year period in older adults. A single nucleotide polymorphism in the COMT (Val158/108Met) gene affects the concentration of dopamine in the prefrontal cortex. In addition, a Val/Met substitution in the pro-domain for BDNF (Val66Met) affects the regulated secretion and trafficking of BDNF with Met carriers showing reduced secretion and poorer cognitive function. We found that impairments over the 10-year span on a task-switching paradigm did not vary as a function of the COMT polymorphism. However, for the BDNF polymorphism the Met carriers performed worse than Val homozygotes at the first testing session but only the Val homozygotes demonstrated a significant reduction in performance over the 10-year span. Our results argue that the COMT polymorphism does not affect the trajectory of age-related executive control decline, whereas the Val/Val polymorphism for BDNF may promote faster rates of cognitive decay in old age. These results are discussed in relation to the role of BDNF in senescence and the transforming impact of the Met allele on cognitive function in old age.
\end{abstract}

Keywords: BDNF, COMT, aging, task-switching, cognition, executive control, longitudinal

\section{INTRODUCTION}

Old age is often accompanied by cognitive impairment with the largest deficits on executive control tasks that are reliant on prefrontal cortex function (Hedden and Gabrieli, 2004). Evidence from both humans and non-human animals suggests that some cognitive deficits observed in old age could be related to disruptions in the dopaminergic and neurotrophic systems (Bäckman et al., 2006; Pang and Lu, 2004). For this reason, genetic polymorphisms that affect the concentration or secretion of neurotrophic factors and neurotransmitters could contribute to some of the individual differences in cognitive function in older adults (de Frias et al., 2004; Harris et al., 2006).

*Correspondence: Kirk I. Erickson, Beckman Institute for Advanced Science and Technology, University of Illinois at Urbana-Champaign, 405 North Matthews Avenue, Urbana, IL 61801, USA. e-mail: kiericks@uiuc.edu

Received: 23 June 2008; paper pending published: 18 August 2008; accepted: 31 August 2008; published online: 23 September 2008.

Citation: Front. Hum. Neurosci. (2008) 2: 11. doi: 10.3389/neuro.09.011.2008

Copyright (c) 2008 Erickson, Kim, Suever, Voss, Francis and Kramer. This is an openaccess article subject to an exclusive license agreement between the authors and the Frontiers Research Foundation, which permits unrestricted use, distribution, and reproduction in any medium, provided the original authors and source are credited.
A functional single nucleotide polymorphism (SNP) in the catechol-O-methyltransferase (COMT) gene mapped to chromosome $22 \mathrm{q} 11$ is thought to influence dopamine concentration in the prefrontal cortex (Akil et al., 2003; Chen et al., 2004; Lachman et al., 1996). COMT is a post-synaptic enzyme that catabolizes dopamine released in the prefrontal cortex, and a valine (Val) to methionine (Met) amino acid substitution at the $158 / 108$ locus of the peptide sequence affects the thermostability of the enzyme. The Met/Met form of this polymorphism produces a less active enzyme resulting in higher dopamine levels than the Val/Val or the Val/Met polymorphism. In schizophrenic populations, as well as normal functioning young adults, the Met/Met form of the COMT polymorphism has been related to superior performance on a number of tests of executive function including the Wisconsin Card Sort Task (Egan et al., 2001; Joober et al., 2002; Malhotra et al., 2002) and the n-back task (Goldberg et al., 2003). In addition, people with the Met/Met form of the COMT polymorphism can elicit higher or lower levels of activity in the prefrontal cortex depending on the task characteristics and cognitive demands (Bertolino et al., 2006; Caldu et al., 2007; Egan et al., 2001; Ettinger et al., 2008; Mattay et al., 2003; Winterer et al., 2006). However, some studies, including a recent meta-analysis have reported that 
the Met/Met form of the COMT polymorphism is not always associated with enhanced or more efficient cognitive function or prefrontal activity compared to Val carriers (Barnett et al., 2008; Bruder et al., 2005; Ho et al., 2005; MacDonald et al., 2007; Tsai et al., 2003).

A few studies have examined whether individual differences in cognitive function in non-demented older adults could be attributed to the COMT polymorphism. One study found no association between the COMT polymorphism and cognitive function (O'Hara et al., 2006) while others have reported better cognitive performance in Met homozygotes compared to carriers of the Val allele (de Frias et al., 2004; Liu et al., 2008). On the other hand, some studies have reported better cognitive function in the Val/Met heterozygotes than either of the homozygotes (Harris et al., 2005), and yet others have reported that both Val/Met heterozygotes and Met homozygotes perform better than the Val/Val counterparts (Starr et al., 2007). Some have speculated that this variation in the literature could be related to (a) an age-related shift in the U-shaped curve that reflects dopamine signaling in the prefrontal cortex (Harris et al., 2005; Starr et al., 2007), (b) that COMT interactions with gender and age (O'Hara et al., 2006) could be masking or driving certain effects, or (c) the tasks that are employed in some studies do not adequately and specifically reflect dopamine or prefrontal cortex engagement and therefore do not validly reflect the impact that the polymorphism has on prefrontal function (O'Hara et al., 2006). There are also a number of other explanations for the discrepancies found between studies including interactions or covariation between the COMT polymorphism and lifestyle factors, demographic variables, or other genes or polymorphisms.

Most of the studies described above have utilized crosssectional designs, that is, they assess the relationship between the COMT polymorphism and cognitive function at one point in time. This method is capable of assessing whether any association exists between the COMT polymorphism and cognition but cannot determine if the polymorphism accounts for withinsubject change in cognitive function across the lifespan. In order to assess whether the polymorphism relates to the trajectory of cognitive decline in old age, longitudinal investigations are needed. Two longitudinal studies have examined the effects of the COMT polymorphism on the trajectory of cognitive decline in older adults. The first study reported that Met homozygotes between 50 and 60 years old experienced a more rapid decline in episodic memory performance over a 5-year period than Val carriers of the same age (de Frias et al., 2004). However, the COMT polymorphism did not moderate changes in episodic memory in either middle-aged adults or adults between 65 and 80 years of age. The main conclusion was that change in episodic memory over a 5-year period is largely independent of the COMT polymorphism except in younger-old adults, that is people between 50 and 60 years of age. A second longitudinal study, with a follow-up period of 4 years, reported that there was no interaction between the COMT polymorphism and time on cognitive function in people between 60 and 64 years of age. However, there was a significant effect of the COMT polymorphism after controlling for general cognitive function at age 11, suggesting that there was a change in the effect that the polymorphism had on cognitive function over the lifespan (Starr et al., 2007). These results suggest that if the effect of the COMT polymorphism on cognitive performance changes as a function of age, the changes occur before the age of 60 .
Although the dopaminergic system has been proposed to underlie some of the age-related cognitive deficits in prefrontal function (Bäckman et al., 2006), it is likely that changes in the concentration or efficacy for an array of molecules and receptors influences cognitive function in old age. For example, brain-derived neurotrophic factor (BDNF) is another molecule involved in cognition that may be related to cognitive impairment and dementia. For example, the mature form of the BDNF (mBDNF) molecule enhances learning and memory and longterm potentiation (Pang et al., 2004), induces synaptic plasticity (Lu, 2003), and promotes neurogenesis (Pencea et al., 2001) and there is evidence that age-related cognitive impairments might be related to a decrease in the production or secretion of BDNF (Hayashi et al., 2001; Pang and Lu, 2004).

A functional polymorphism in the gene for BDNF produces a single amino acid substitution of Val to Met at codon 66 in the pro-domain, with the Met allele selectively impairing the regulated secretion and intracellular trafficking of BDNF in primary cortical neurons and neurosecretory cells (Chen et al., 2004; Egan et al., 2003). Previous studies have found that people with the Met allele have impaired episodic memory, working memory, and hippocampal function (Egan et al., 2003; Hariri et al., 2003; Ho et al., 2006) and lower hippocampal levels of $N$-acetylaspartate, a putative measure of neuronal integrity (Egan et al., 2003). In addition, Met carriers have less gray matter volume throughout the prefrontal and middle temporal lobes compared to Val carriers (Ho et al., 2006, 2007; Pezawas et al., 2004).

Only a few studies have examined the relationship between the BDNF polymorphism and age-related cognitive impairment. Inconsistent with the majority of studies in young adults and patients with depression, Met homozygotes at 64 and 79 years of age outperformed the Val homozygotes and heterozygotes (Harris et al., 2006) after controlling for sex and cognitive performance at age 11. This finding suggests that the influence that BDNF has on cognitive function may change across the lifespan and that the Met allele may be neuroprotective during later stages of life. Consistent with this finding, there is some evidence that the Val allele may increase the risk for Alzheimer's disease (Matsushita et al., 2005; Ventriglia et al., 2002), but some recent studies have failed to find such a relationship (Akatsu et al., 2006; He et al., 2007). Others however have reported the opposite finding, that is older adults carrying the Met allele perform worse across a variety of cognitive domains compared with the Val homozygotes (Miyajima et al., 2008) and have a higher risk for developing late-life depression (Taylor et al., 2007) and white-matter hyperintensities (Taylor et al., 2008). However, similar to the research on COMT, the studies mentioned above on BDNF have been cross-sectional in nature and are therefore limited in their capability for drawing conclusions concerning the relationship between within-subject changes in cognitive function and the BDNF polymorphism. A longitudinal study would help resolve these issues.

The current study assessed whether cognitive decline over a 10 -year span in a group of older adults was moderated by the COMT and/or BDNF polymorphism. Our within-subject design provided us with more statistical power than previously conducted cross-sectional studies. To assess whether individual differences in cognitive decline varied as a function of the BDNF or COMT polymorphism we utilized a well-studied task-switching paradigm that requires participants to rapidly switch between one simple cognitive task to a different cognitive task (Rogers and Monsell, 1995). This paradigm was chosen because of its 
established capability to tap prefrontal and executive resources (Braver et al., 2003; Kimberg et al., 2000) and to demonstrate age-related deficits (Kramer et al., 1999a; Kray and Lindenberger, 2000). Therefore, this paradigm allowed us to test the prediction that (a) the COMT and BDNF polymorphisms affect performance on tasks that depend on prefrontal function, and (b) agerelated executive deficits vary as a function of the BDNF or COMT polymorphism. Furthermore, our 10-year longitudinal design doubles the length of previous longitudinal studies to date. A long span between testing periods increases the likelihood of finding changes in cognitive function across time, which then provides us with the variation necessary to assess whether the COMT and/or BDNF polymorphisms moderates the decline in performance. Based on the extant literature, we predicted that the COMT polymorphism would explain little of the changes in cognitive performance over the 10-year span given that the age of our sample at the first time point was on average above the age in which interactions across the lifespan have been observed in prior investigations (de Frias et al., 2004). On the other hand, there have not been any longitudinal studies examining whether the BDNF polymorphism influences cognitive function across the adult lifespan. Some have argued that at younger ages, the Val/Val allelic combination provides some neuronal and cognitive benefits, but with advancing age the Met allele, instead of being detrimental to cognitive and brain function and morphology, actually carries some protection against the development of dementia and cognitive impairment (Harris et al., 2006). We explored these hypotheses in the current study.

\section{MATERIALS AND METHODS \\ PARTICIPANTS}

Fifty-three healthy older adults (14 male, mean age of $75.5 \pm 5.3$, range: $67-86$ ) who had participated in a previous study $\sim 10$ years ago (Kramer et al., 1999b) were recruited to participate in this study. Forty-three percent of the original sample of 124 participants agreed to return. We assessed whether the participants who agreed to return for the follow-up session were different from those who declined the invitation to return for the follow-up session in terms of male to female ratio or age. The male to female ratio was similar between those that agreed to return $(\mathrm{m}: \mathrm{f}=0.35)$ versus those that declined the invitation to return $(\mathrm{m}: \mathrm{f}=0.38)$, with slightly more men declining the invitation to return for the follow-up. Furthermore, the average age of the participants was nearly equivalent for those that returned [average age $=65.33(10$ years ago $)]$ compared with those that declined to return for the follow-up session [average age $=65.50$ (10 years ago)]. Independent samples $t$-tests demonstrated that these differences were not significant (all $p>0.05$ ). The University of Illinois Institutional Review Board approved the study, and all volunteers signed an informed consent.

\section{TASK-SWITCHING}

The task-switching paradigm examines subjects' ability to rapidly disengage from the performance of one task and switch to another. Subjects performed two different tasks that alternated after every two trials - two trials of one task followed by two trials of the other task and so on (see Figure 1). In one task subjects performed an odd/even numerical judgment (i.e., is a single digit number odd or even). In the other task subjects performed a vowel/consonant judgment. When one type of trial (e.g., digit judgment) was followed by a trial of the same type (e.g., digit judgment) it was labeled a Repeat trial. However, when participants needed to respond to a trial that was of a different task type than the previous trial, it was labeled as a Switch. Response times (RTs) to switch trials are higher than RTs to repeat trials and the accuracy rates are lower.

\section{Task-Switching}

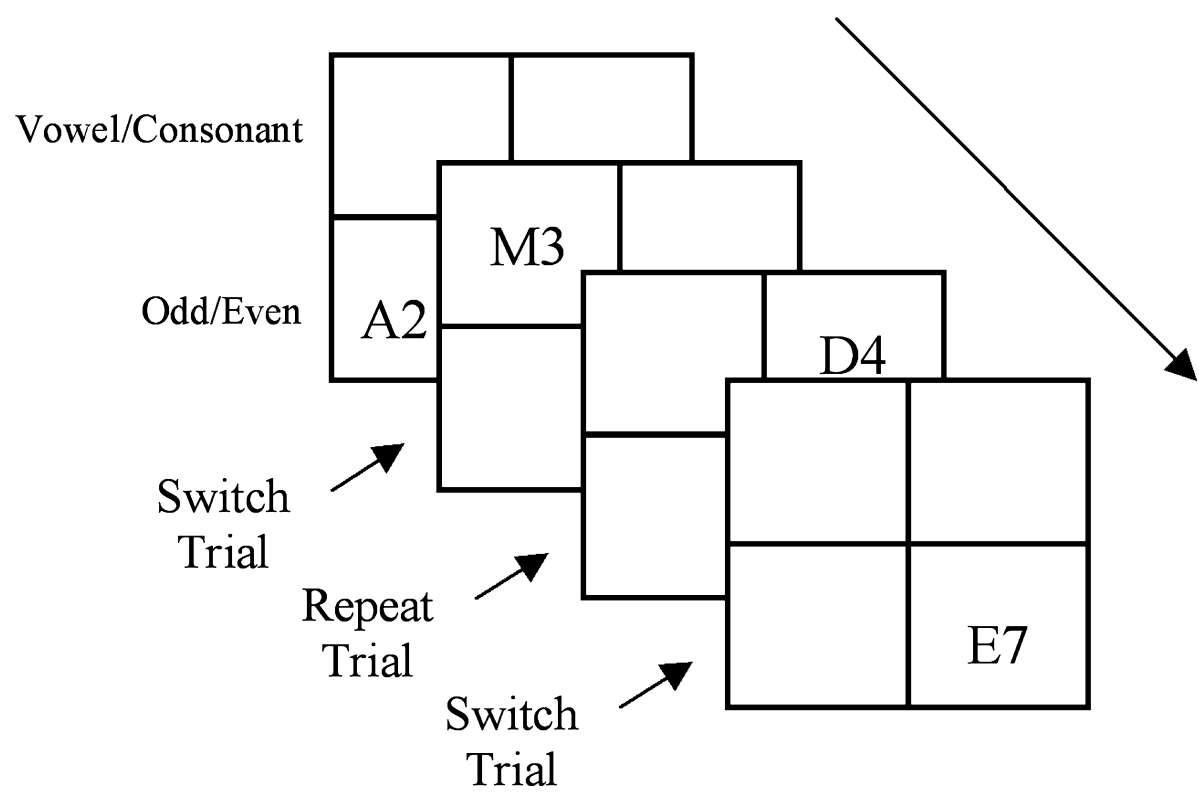

Figure 1 | Description of the task-switching paradigm. Letter and digit stimuli were presented simultaneously in a $2 \times 2$ grid and participants had to switch between responding to the letters versus responding to the numbers on every 3rd trial (adapted from Rogers and Monsell, 1995). 
The task stimuli, a letter and a single digit number, were presented together in a $2 \times 2$ matrix centered in the middle of the computer screen. When the letter and digit were located in one half of the matrix subjects performed the odd/even judgment task, when the letter and digit were in the other half of the matrix subjects performed the consonant/vowel judgment task (e.g., perform odd/even judgment for upper two quadrants of the matrix and consonant/vowel judgment for lower two quadrants). The letter and digit were presented in the matrix in a continuous clockwise direction. Thus, the occurrence of a task switch was predictable. The location (i.e., left, right, upper, lower) of each task was counterbalanced across subjects (task adapted from Rogers and Monsell, 1995).

Each stimulus pair was presented until the subject responded and then the next stimulus pair was presented $400 \mathrm{~ms}$ following response. Subjects responded with one of two keys on the computer keyboard for both of the tasks (e.g., one key was used to respond to an odd number or a consonant). The key representations were counterbalanced across subjects.

Subjects first performed two 30 trial single task blocks followed by one 30 trial task-switching block as practice. The practice blocks were then followed by four 60 trial task-switching blocks. The main dependent variables included mean RT and accuracy to both Repeat and Switch conditions.

\section{MMSE AND IQ}

To test for general cognitive function and possible dementia we employed a modified and revised version of the Mini-Mental Status Examination (MMSE) that has a high score of 57 and a cut-off for possible dementia at 51. To assess the relationship between the BDNF and COMT polymorphisms and general intelligence (IQ) we employed the Kaufman Brief Intelligence Test. Both of these tests were conducted at the Time 2 session and were therefore not subjected to a repeated-measures analysis to assess for change in either score.

\section{GENOTYPING}

Buccal cells were collected from all participants using MasterAmp ${ }^{\mathrm{TM}}$ Buccal Swab Brushes (Epicentre Biotechnologies). Genomic deoxyribonucleic acid (DNA) was extracted from the buccal swabs using MasterAmp ${ }^{\mathrm{TM}}$ DNA Extraction Solution (Epicentre Biotechnologies).

\section{COMT}

Primers COMT-F 5'-TCA CCA TCG AGA TCA ACC CC- $3^{\prime}$ and COMT-R 5'-GAA CGT GGT GTG AAC ACC TG-3' were used to amplify the 176 bp polymorphic COMT fragment (Barr et al., 1999). The amplification was done in $50 \mu \mathrm{l}$ reactions containing $\sim 125 \mathrm{ng}$ genomic DNA, $200 \mu \mathrm{M}$ deoxynucleoside triphosphates (dNTPs), $10 \mathrm{pmol} / \mathrm{l}$ of each primer, $10 \times$ HotStarTaq $^{\circledR}$ buffer (QIAGEN), and 1 U HotStarTaq ${ }^{\circledR}$ DNA polymerase (QIAGEN). Polymerase chain reaction (PCR) conditions consisted of an initial denaturation step at $95^{\circ} \mathrm{C}$ for $15 \mathrm{~min}$ followed by 30 cycles on a thermocycler (denaturation at $94^{\circ} \mathrm{C}$ for $30 \mathrm{~s}$, annealing at $52^{\circ} \mathrm{C}$ for $30 \mathrm{~s}$, and extension at $72^{\circ} \mathrm{C}$ for $30 \mathrm{~s}$ ) and finished with a final extension at $72^{\circ} \mathrm{C}$ for $10 \mathrm{~min}$. Eight microliters of the PCR product were digested with $10 \mathrm{U}$ NlaIII (New England Biolabs) (Barr et al., 1999) at $37^{\circ} \mathrm{C}$ for $1 \mathrm{~h}$ and analyzed by gel electrophoresis on a $3.5 \%$ MetaPhor $^{\circledR}$ agarose gel (Cambrex Bioscience, Inc./Lonza). The gel was immersed in an ethidium bromide solution for $15 \mathrm{~min}$ and visualized under ultraviolet light. Digestion resulted in bands of 82,54 , and $41 \mathrm{bp}$ for the
$\mathrm{Val}^{158}$ allele. The $82 \mathrm{bp}$ fragment was cut into 64 and 18 bp bands for the Met ${ }^{158}$ allele.

\section{BDNF}

Primers BDNF-F $5^{\prime}$-GAG GCT TGA CAT CAT TGG CT- ${ }^{\prime}$ and BDNF-R 5'-CGT GTA CAA GTC TGC GTC CT- ${ }^{\prime}$ ' were used to amplify the $113 \mathrm{bp}$ polymorphic BDNF fragment (Neves-Pereira et al., 2002). The amplification was done in $25 \mu \mathrm{l}$ reactions containing $\sim 125$ ng genomic DNA, $200 \mu \mathrm{M}$ dNTPs, 10 pmol of each primer, $1.5 \mathrm{mM} \mathrm{MgCl}_{2}, 1 \mathrm{U}$ Taq DNA polymerase (Invitrogen) (adapted from Neves-Pereira et al., 2002). PCR conditions consisted of an initial denaturation step at $95^{\circ} \mathrm{C}$ for $5 \mathrm{~min}$ followed by 30 cycles on a thermocycler (denaturation at $94^{\circ} \mathrm{C}$ for $30 \mathrm{~s}$, annealing at $60^{\circ} \mathrm{C}$ for $30 \mathrm{~s}$, and extension at $72^{\circ} \mathrm{C}$ for $30 \mathrm{~s}$ ) and finished with a final extension at $72^{\circ} \mathrm{C}$ for $5 \mathrm{~min}$ (Neves-Pereira et al., 2002). $6.5 \mu \mathrm{l}$ of the PCR product were digested with $3 \mathrm{U}$ Eco721 (Fermentas) at $37^{\circ} \mathrm{C}$ overnight and analyzed by gel electrophoresis on a 4\% 3:1 NuSieve ${ }^{\circledR}$ agarose gel (Cambrex Biosciences, Inc./Lonza) (adapted from Neves-Pereira et al., 2002). The gel was immersed in an ethidium bromide solution for $10 \mathrm{~min}$ and visualized under ultraviolet light. Digestion resulted in an uncut band of $113 \mathrm{bp}$ for the Met ${ }^{66}$ allele. The $113 \mathrm{bp}$ fragment is cut into 78 and $35 \mathrm{bp}$ bands for the $\mathrm{Val}^{66}$ allele.

\section{STATISTICAL ANALYSIS}

We analyzed the task-switching data (reaction time and accuracy) with repeated-measures analyses of variance with Time (1997, 2007) and Condition (repeat, switch) as within-subject factors and group (COMT or BDNF genotype) as a between-subjects factor. Effect sizes were calculated and reported here as partial etasquared $\left(\eta_{\mathrm{p}}^{2}\right)$. IQ scores were used as a covariate for COMT (see 'Results' section). One-way ANOVAs and independent samples $t$-tests were also employed to assess differences in demographic characteristics, IQ, MMSE scores, or task-switching performance as a function of the BDNF and COMT polymorphism at separate time points. All data was analyzed using SPSS 16.02 for Mac.

\section{RESULTS \\ COMT \\ Demographics}

One-way ANOVAs were used to test whether the COMT SNP was related to age or IQ (see Table 1 ). We found no effect of age $\left[F(2,52)=1.87 ;\right.$ n.s; $\left.\eta_{p}^{2}=0.07\right]$, but we did find a trend for an effect of IQ obtained from the Kaufman Brief Intelligence Scale $\left[F(2,52)=2.61 ; p<0.08 ; \eta_{p}^{2}=0.09\right]$. Post hoc tests revealed that those with the Val/Val form of the COMT gene had lower IQ scores than the heterozygotes $(p<0.04)$ and marginally lower scores than the Met homozygotes $(p<0.06)$. Met homozygotes and Val/Met heterozygotes did not differ in IQ scores $(p<0.92)$.

\section{MMSE}

We employed a one-way ANOVA to examine whether the COMT SNP was related to performance on the MMSE task - a general and widely used measure to test for possible dementia and impaired cognitive function (see Table 1). There was no relationship between the COMT SNP and performance on the $\operatorname{MMSE}\left[F(2,51)=1.84 ;\right.$ n.s.; $\left.\eta_{\mathrm{p}}^{2}=0.07\right]$.

\section{Task-switching}

Repeated-measures ANOVAs were run with COMT genotype (Val/Val; Val/Met; Met/Met) as a between-subjects factor and 
Table 1 | Demographic information, IQ, and MMSE scores are stratified by both the COMT and BDNF polymorphism.

\begin{tabular}{|c|c|c|c|c|c|}
\hline & $N$ & Sex & Age (Time 1) & IQ & MMSE \\
\hline \multicolumn{6}{|l|}{ COMT } \\
\hline $\mathrm{Val} / \mathrm{Val}$ & 16 & $11 \mathrm{~F} / 5 \mathrm{M}$ & $65.06(5.60)$ & 109.25 (14.09) & $52.12(5.29)$ \\
\hline Val/Met & 19 & $16 \mathrm{~F} / 3 \mathrm{M}$ & $68.16(5.22)$ & $116.16(8.25)$ & $53.42(2.61)$ \\
\hline Met/Met & 18 & $12 \mathrm{~F} / 6 \mathrm{M}$ & $65.56(4.68)$ & $115.83(6.28)$ & $54.59(2.78)$ \\
\hline \multicolumn{6}{|l|}{ BDNF } \\
\hline $\mathrm{Val} / \mathrm{Val}$ & 29 & $23 \mathrm{~F} / 6 \mathrm{M}$ & $65.83(5.47)$ & $112.10(10.45)$ & $52.75(4.44)$ \\
\hline Val/Met & 24 & 16F/8M & $66.96(5.02)$ & $116.21(9.56)$ & $54.17(2.61)$ \\
\hline Met/Met & 0 & - & - & - & - \\
\hline
\end{tabular}

Standard deviations are represented in parentheses. There was a trend for IQ to vary as a function of COMT genotype and the Val/Val form of the polymorphism reliably differed from the Val/Met form.

Table 2 | Mean response times and accuracy rates for repeat and switch conditions for both BDNF and COMT polymorphisms.

\begin{tabular}{|c|c|c|c|c|c|c|c|c|}
\hline & \multicolumn{4}{|c|}{ Time 1} & \multicolumn{4}{|c|}{ Time 2} \\
\hline & \multicolumn{2}{|c|}{ Repeat } & \multicolumn{2}{|c|}{ Switch } & \multicolumn{2}{|c|}{ Repeat } & \multicolumn{2}{|c|}{ Switch } \\
\hline & $\mathbf{R T}$ & ACC & $\mathbf{R T}$ & ACC & RT & ACC & RT & ACC \\
\hline \multicolumn{9}{|l|}{ COMT } \\
\hline $\mathrm{Val} / \mathrm{Val}$ & $1607.81(585.45)$ & $93.4(6.9)$ & 2402.61 (735.38) & $95.6(7.2)$ & 1761.99 (831.38) & $87.5(18.5)$ & 2511.97 (1330.52) & $82.0(18.9)$ \\
\hline Val/Met & 1384.06 (488.63) & $96.3(6.9)$ & 2120.85 (733.84) & $94.3(5.3)$ & $1474.34(344.05)$ & $93.8(10.1)$ & 2311.68 (556.09) & $89.2(13.2)$ \\
\hline Met/Met & $1271.42(342.84)$ & $99.5(.66)$ & 1912.09 (671.48) & $98.0(1.5)$ & $1623.44(583.15)$ & $93.7(11.5)$ & 2221.41 (917.41) & $90.6(12.4)$ \\
\hline \multicolumn{9}{|l|}{ BDNF } \\
\hline $\mathrm{Val} / \mathrm{Val}$ & 1262.56 (287.31) & $97.7(4.9)$ & 1902.27 (552.69) & $95.9(5.5)$ & $1680.62(640.42)$ & $95.1(4.7)$ & 2471.60 (981.44) & $89.2(9.8)$ \\
\hline Val/Met & $1536.32(588.06)$ & $97.4(6.2)$ & 2329.38 (822.82) & $96.1(4.5)$ & $1515.12(507.37)$ & $89.1(17.8)$ & 2163.52 (825.08) & $86.7(18.5)$ \\
\hline
\end{tabular}

Standard deviations are represented in parentheses.

Time (1997 - Time 1; 2007 - Time 2) and Condition (Repeat; Switch) as within-subjects factors on RTs and accuracy rates separately. We included IQ score as a covariate given its marginal relationship to the COMT polymorphism (see above). Therefore, all results described here can be considered to be statistically independent from IQ.

We found that main effects of Time $[F(1,42)=1.75 ;$ n.s. $]$ and Genotype $[F(2,42)=1.23$; n.s. $]$ were not significant (Table 2 ). Furthermore, consistent with our hypotheses and results from previous studies (de Frias et al., 2004; Harris et al., 2005) we failed to find a Time $\times$ Genotype interaction $[F(2,42)=0.34$; n.s.; $\left.\eta_{p}^{2}=0.02\right]$ or a Time $\times$ Genotype $\times$ Condition interaction on the RTs from the task-switching paradigm $[F(2,42)=0.30$; n.s.; $\eta_{p}^{2}=0.01$ ] indicating that RTs (for both the repeat and switch conditions) did not change over the 10-year period as a function of the COMT polymorphism. Converting the RTs into a switch cost (switch RT - repeat RT) at each time point confirmed this effect. We also conducted the same repeatedmeasures analysis on the accuracy rates and found neither a significant Time $\times$ Genotype interaction $[F(2,42)=0.86$; n.s.; $\left.\eta_{\mathrm{p}}^{2}=0.04\right]$ nor a Time $\times$ Genotype $\times$ Condition interaction $\left[F(2,42)=0.70 ;\right.$ n.s.; $\left.\eta_{p}^{2}=0.03\right]$.

To assess performance on the task-switching paradigm at individual time points as a function of the COMT polymorphism we conducted a series of univariate ANOVAs with Genotype as a fixed factor and RTs and accuracy rates for each condition as the dependent variable. There was no effect of Genotype on the RTs for either the switch condition or the repeat condition at either time point (all $p>0.05$; all $\eta_{p}^{2}<0.07$ ). However, for the accuracy measures, we found that at Time 1 there was a marginally significant effect of Genotype for the switch condition $[F(2,51)=2.85$; $\left.p<0.06 ; \eta_{\mathrm{p}}^{2}=0.11\right]$. Post hoc comparisons revealed that the Met homozygotes had significantly higher accuracy rates compared with the heterozygotes $(p<0.02)$, but were not reliably different from the Val homozygotes $(p<0.63)$. No other comparisons reached significance (all $p>0.05$ ).

There were six participants who could not complete the task-switching paradigm at Time 2 because the task was too challenging, however these participants had been able to successfully complete the task 10 years prior. Interestingly, five out of the six participants had the Val/Val form of the COMT polymorphism and the other participant was heterozygous. None of the participants who failed to complete the task at Time 2 were homozygous for the Met/Met form. We tested whether this distribution was significantly different from chance using a $\chi^{2}$ non-parametric test with the respective sample sizes for each polymorphism as the null hypothesis (Val/Val $=16$, Val/ Met $=19$, Met/Met $=18)$. We found a non-significant $\chi^{2}(1.05$; $p<0.59)$ for the given frequencies, indicating that although there were more Val homozygotes who failed to complete the task, the number of participants who fell into this category was not significantly greater than chance. However, studies with larger samples could more validly test this trend.

\section{BDNF \\ Demographics}

We conducted independent samples $t$-tests to assess whether any demographics variables were related to the BDNF polymorphism (see Table 1$)$. Neither age $[t(1,51)=0.78$; n.s.] nor IQ $[t(1,51)=1.48 ;$ n.s. $]$ was related to the BDNF polymorphism. 


\section{MMSE}

In an independent samples $t$-test we found that there was no relationship between the BDNF polymorphism and performance on the MMSE $[t(1,51)=1.42 ;$ n.s. - see Table 1$]$.

\section{Task-switching}

Similar to the COMT polymorphism described above, we assessed the influence of the BDNF polymorphism on cognitive decline in the task-switching paradigm by employing a repeatedmeasures ANOVA with Genotype (Val/Val; Val/Met) as a betweensubjects factor and Time (1997 - Time 1; 2007 - Time 2) and Condition (Repeat; Switch) as within-subjects factors.

For BDNF, we found that main effects of Time $[F(1,44)=$ 2.13 ; n.s. $]$ and Genotype $[F(1,44)=0.09$; n.s.] were not significant (Table 2). However, consistent with the view that the
Met allele might provide some protection in old age or that the Val homozygotes might experience greater decline with advancing age, we found a significant Time $\times$ Genotype interaction $\left[F(1,44)=7.54 ; p<0.009 ; \eta_{p}^{2}=0.15\right]$ on RTs in the taskswitching paradigm such that the Val homozygotes experienced a significantly greater decline in performance over the 10 -year period compared to the Met carriers (see Figure 2). There was also a trend for a Time $\times$ Genotype $\times$ Condition interaction $\left[F(1,44)=3.15 ; p<0.08 ; \eta^{2}=0.07\right]$ such that Val carriers experienced a greater decline in performance for the switch condition compared with the repeat condition over the 10-year span compared to the Met carriers. This trend was confirmed by examining switch cost (switch RT - repeat RT). For the accuracy measures, neither the Time $\times$ Genotype interaction or the Time $\times$ Genotype $\times$ Condition interaction reached significance
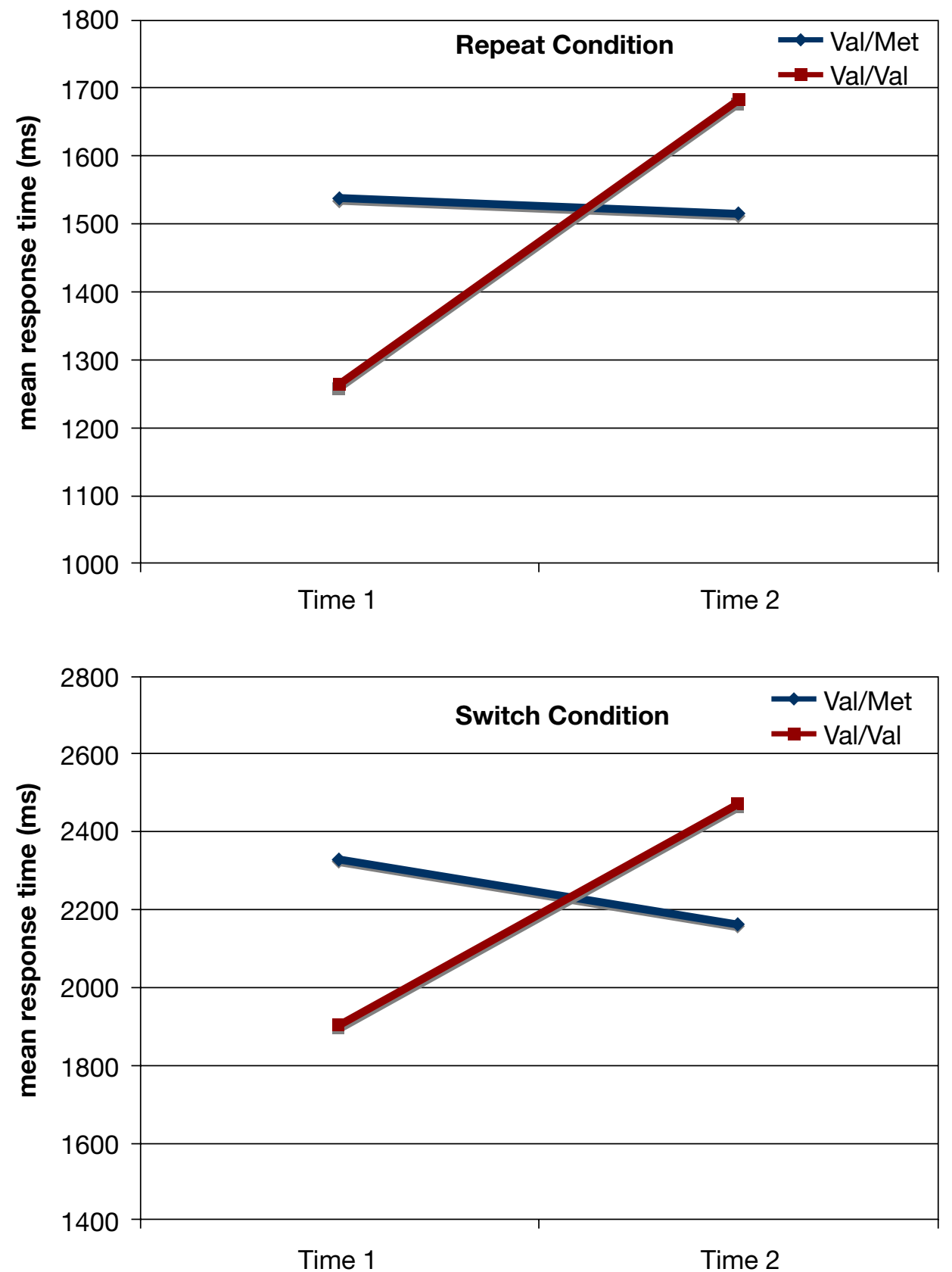

Figure 2 | Results from the task-switching paradigm as a function of the BDNF polymorphism and Time for each condition. Only the Val/Val homozygotes experienced a decline in performance for both the repeat and switch conditions, whereas the Val/Met carriers showed no decline over the 10-year span. 
indicating that unlike the RTs, the accuracy rates were not influenced by the BDNF polymorphism. However, we found a significant Genotype $\times$ Condition interaction on the accuracy rates $\left[F(1,44)=4.94 ; p<0.03 ; \eta_{\mathrm{p}}^{2}=0.10\right]$ with the Val homozygotes performing better than the heterozygotes on the repeat condition compared to the switch condition.

We conducted a series of independent $t$-tests at each time point and for each condition separately to test whether the two groups differed at either time point. There were no significant differences between the two BDNF polymorphisms at either time point for RTs or accuracy measures (all $p>0.05$ ).

Like the COMT polymorphism results described above we examined whether the participants who could not complete the task-switching paradigm at Time 2 had a particular form of the BDNF polymorphism. The results from the $\chi^{2}$ test was not significant $(0.04 ; p<0.83)$ indicating that the BDNF polymorphism did not explain the failure to complete the task-switching paradigm at Time 2 .

\section{DISCUSSION}

In this study we examined whether the BDNF or COMT polymorphisms could explain variation in the trajectory of cognitive decline over a 10-year span in older adults. Our participants were older than the participants in a previous 5-year longitudinal study examining the influence of the COMT polymorphism on cognitive performance in older adults (de Frias et al., 2004). Furthermore, this is the first known longitudinal study of the effects of the BDNF polymorphism on age-related cognitive decline.

Consistent with prior studies, we found no evidence that the COMT polymorphism contributes to age-related declines in executive function as assessed by the task-switching paradigm (O'Hara et al., 2006; Starr et al., 2007). In a longitudinal study, an interaction with age was only reported for people between 50 and 60 years of age (de Frias et al., 2004). The participants in our sample were 65 years of age on average in 1997 and $\sim 75$ years of age as of 2007 , and therefore may have been outside the age range to detect an interaction if such an interaction is specific to the 5th decade of life (de Frias et al., 2004). Our results are more consistent with a 4-year longitudinal study of older adults that did not find an interaction between the COMT polymorphism and age on cognitive function (Starr et al., 2007). In short, our results suggest that, after the 6th decade of life, the COMT polymorphism does not explain cognitive decline over a 10 -year period.

On the other hand, the BDNF polymorphism reliably explained variation in age-related decline in performance for both the repeat and switch conditions of the task-switching paradigm (Figure 2). In a cross-sectional study, Harris et al. (2006) reported that older adult Met homozygotes had better reasoning skills than Val homozygotes or heterozygotes. Partially consistent with this finding, we demonstrate that the Met carriers have spared cognitive function over a 10 -year period while the Val homozygotes showed a significant decline in performance. These results, however, are generally inconsistent with the majority of the BDNF-gene literature, which typically reports poorer performance and functioning for Met carriers in both young and old adults (Egan et al., 2003; Taylor et al., 2007). This discrepancy between our finding and cross-sectional studies might be explained by the age of the sample studied. Based on our longitudinal results it is apparent that at an average age of
65 the Val homozygotes tend to perform better than Met carriers, however there is a crossover such that by the average age of 75 Val homozygotes tend to perform worse than Met carriers. Cross-sectional studies that assess older adults around 65 years of age might find cognitive enhancement associated with the Val/Val genotype, whereas studies examining a sample with an average age of 75 might produce the opposite pattern.

There are a number of possible reasons that could explain the crossover effect that we observed. First, we have no information regarding the trajectory of cognitive decline earlier in life. Therefore, it is possible that Met carriers could have shown a decline in performance at an earlier age and then plateaued, while the Val homozygotes had spared cognitive function until the 6 th to 7 th decade of life.

Second, a number of factors that influence BDNF signaling could be altered during old age such that greater secretion of BDNF would be detrimental for cognitive and neural activity. For example, the precursor form of the BDNF (pro-BDNF) molecule and the mBDNF have distinct receptors and signaling cascades resulting in opposing effects on the nervous system (Lu et al., 2005; Pang et al., 2004). Pro-BDNF enhances the capability for eliciting long-term depression, synaptic retraction, and cell death; whereas mBDNF increases the capability for eliciting long-term potentiation, synaptic formation, and cell survival (see Lu et al., 2005). In neurons, pro-BDNF is usually converted to the mature form in the extracellular space by proteases including tissue plasminogen activator (tPA). Cerebral levels of tPA decline with age and this decline is exacerbated in rodent models of Alzheimer disease (Cacquevel, et al., 2007). Therefore, greater secretion of BDNF may not enhance cognitive and neuronal function unless the cleavage molecules are present to convert it from its precursor form to its mature form. In fact, greater secretion of pro-BDNF into the synaptic space without an adequate concentration of cleavage molecules (e.g., tPA) to convert it to its mature form might result in cognitive impairment and decline instead of cognitive enhancement (Lu et al., 2005; Pang et al., 2004). The Val/Val form of the BDNF polymorphism increases the regulated secretion and trafficking of the pro-BDNF molecule (Egan et al., 2003), and we find that this polymorphism is associated with a more rapid decline in cognitive function than their genetic counterparts that have reduced secretion of proBDNF. Our result is clearly in line with the hypothesis that the enhancing role of BDNF on cognition is dependent on a number of molecular factors including those that influence the presence or concentration of the cleavage molecules and that these supporting molecules might also be affected by aging. In short, a complex array of molecules are involved in BDNF signaling, and increased cellular secretion due to a genetic polymorphism may not always be associated with better function.

Third, a number of environmental factors influence BDNF translation and concentration in rodents including environmental enrichment (van Praag et al., 2000), physical exercise (Cotman et al., 2007), caloric restriction (Mattson et al., 2003), and estrogen administration (Scharfman and Maclusky, 2005). Interactions between the BDNF polymorphism and any of these environmental factors could be moderating the age-interaction observed in this study. In short, there are a multitude of reasons for why older adult Met carriers would demonstrate spared cognitive function while Val homozygotes undergo a greater decline in cognitive function with advanced age.

It is also important to note that we did not find that the BDNF polymorphism disproportionately affected one of the 
task-switching conditions more than the other. That is, the switch cost (switch RT - repeat RT) was only marginally related to the BDNF polymorphism $(p=0.08)$. This result suggests that the BDNF polymorphism, and its role in influencing the trajectory of cognitive decline in old age, may primarily affect decline in speed of processing rather than a domain specific decline in executive function. More research employing a wider variety of tasks is warranted to examine this hypothesis.

It is also interesting to consider our results within a cognitive reserve framework in which individuals with more education often demonstrate spared cognitive function despite having disease-related pathology (Fratiglioni and Wang, 2007). We found that BDNF heterozygous individuals that performed more poorly 10 years earlier showed more stability and reserve over the 10-year span. In our sample, IQ scores, which are often used as a measure of cognitive reserve, were unrelated to the BDNF genotype, suggesting that both homozygotes and heterozygotes had equivalent levels of 'reserve' as assessed by this measure. It might be possible that the BDNF genotype acts as a moderator between cognitive reserve measures such as IQ or education and cognitive function. This hypothesis would predict that Val/ Val individuals with higher levels of education or IQ would not show the same rate of decline in performance as Val/Val individuals with lower education or IQ scores. A study with a larger sample size would be more capable of investigating this potential moderating relationship.

Finally, there are a number of limitations of the current study. First, although we have gained statistical power compared to cross-sectional studies by conducting within-subjects comparisons, we have lost statistical power by only being able to recruit 53 out of the 124 original participants. Therefore, our small sample size could have precluded our ability to find a significant interaction with the COMT polymorphism. However, despite this small sample size, we were able to detect a significant effect of the BDNF polymorphism on task-switching performance and our effect sizes were similar to those reported by prior studies (de Frias et al., 2004; Harris et al., 2005, 2006). Second, although we report that the individuals who returned for the follow-up session did not differ in age or sex from those who decline to return, it is possible that the 53 people who agreed to participate in this study were healthier, higher functioning, and may not be a representative sample of the BDNF or COMT polymorphisms in this age range. This potential bias could have affected the pattern of results that we describe here. A longer longitudinal study with a larger sample size would be able to reduce this possible confound.

In sum, we report that the BDNF polymorphism, and not the COMT polymorphism, influences the rate of cognitive decline over a 10-year span in older adults. Both conditions of the taskswitching paradigm were affected by the BDNF polymorphism while general cognitive function as assessed by the MMSE and IQ tests was not related to the BDNF polymorphism. The Met carriers of the BDNF gene demonstrated spared function over the 10-year span while the Val homozygotes experienced a significant decline in performance. This result is inconsistent with a growing literature on the impact of the BDNF polymorphism on depression, cognitive function, and neural activity in young adults, but is partially consistent with at least one study in older adults (Harris et al., 2006). More longitudinal studies with larger sample sizes that employ a wider range of cognitive tests and a more comprehensive array of factors that could explain some individual differences (e.g., physical fitness measurements) and possibly covary or interact with the BDNF and COMT polymorphisms would greatly enhance the interpretation of the results described in this study.

\section{CONFLICT OF INTEREST STATEMENT}

The authors declare that the research was conducted in the absence of any commercial or financial relationships that could be construed as a potential conflict of interest.

\section{ACKNOWLEDGEMENTS}

This work was supported in part by a Seed Grant awarded to Kirk I. Erickson and Jennifer S. Kim from the Center for Healthy Minds, funded through the National Institute on Aging Grant (P30-AG023101) of the National Institutes of Health and grants from the National Institute on Aging (RO1 AG25667 and RO1 AG25302). We would like to thank Brooke Bachelor, Zuha Warraich, Chris Grant, and Edward Malkowski for their help in genotyping and participant recruitment and testing.

\section{REFERENCES}

Akatsu, H., Yamagata, H. D., Kawamata, J., Kamino, K., Takeda, M., Yamamoto, T., et al. (2006). Variations in the BDNF gene in autopsy-confirmed Alzheimer's disease and dementia with Lewy bodies in Japan. Dement. Geriatr. Cogn. Disord. 22, 216-22.

Akil, M., Kolachana, B. S., Rothmond, D. A., Hyde, T. M., Weinberger, D. R., and Kleinman, J. E. (2003). Catechol-O-methyltransferase genotype and dopamine regulation in the human brain. J. Neurosci. 23, 2008-2013.

Bäckman, L., Nyberg, L., Lindenberger, U., Li, S. C., and Farde, L. (2006). The correlative triad among aging, dopamine, and cognition: current status and future prospects. Neurosci. Biobehav. Rev. 30, 791-807.

Barnett, J. H., Scoriels, L., and Munafo, M. R. (2008). Meta-analysis of the cognitive effects of the catechol-O-methyltransferase gene Val158/108Met polymorphism. Biol. Psychiatry 64:137-144. [Epub, PMID: 18339359].

Barr, C. L., Wigg, K., Malone, M., Schachar, R., Tannock, R., Roberts, W., et al. (1999). Linkage study of catechol-O-methyltransferase and attention-deficit hyperactivity disorder. Am. J. Med. Genet. 88, 710-713.

Bertolino, A., Blasi, G., Latorre, V., Rubino, V., Rampino, A., Sinibaldi, L., et al. (2006). Additive effects of genetic variation in dopamine regulating genes on working memory cortical activity in human brain. J. Neurosci. 26, 3918-3922.

Braver, T. S., Reynolds, J. R., and Donaldson, D. I. (2003). Neural mechanisms of transient and sustained cognitive control during task switching. Neuron 39, 713-726.

Bruder, G. E., Keilp, J. G., Xu, H., Shikgman, M., Schori, E., Gorman, J. M., et al. (2005). Catechol-O-methyltransferase (COMT) genoytpes and working memory: associations with differing cognitive operations. Biol. Psychiatry 58, 901-907.

Cacquevel, M., Launay, S., Castel, H., Benchenane, K., Chéenne, S., Buée, L., et al. (2007). Ageing and amyloid-beta peptide deposition contribute to an impaired brain tissue plasminogen activator activity by different mechanisms. Neurobiol. Dis. 27, 164-173.

Caldu, X., Vendrell, P., Bartrés-Faz, D., Clemente, I., Bargalló, N., Jurado, M. A., et al. (2007). Impact of the COMT Val108/158 Met and DAT genotypes on prefrontal function in healthy subjects. Neuroimage 37, 1437-1444.

Chen, J., Lipska, B. K., Halim, N., Ma, Q. D., Matsumoto, M., Melhem, S., et al. (2004). Functional analysis of genetic variation in catechol-O-methyltransferase (COMT): effects on mRNA, protein, and enzyme activity in postmortem human brain. Am. J. Hum. Genet. 75, 807-821.

Chen, Z. Y., Patel, P. D., Sant, G., Meng, C. X., Teng, K. K., Hempstead, B. L., et al. (2004). Variant brain-derived neurotrophic factor (BDNF) (Met66) alters the intracellular trafficking and activity-dependent secretion of wild-type BDNF in neurosecretory cells and cortical neurons. J. Neurosci. 24, 4401-4411.

Cotman, C. W., Berchtold, N. C., and Christie, L. A. (2007). Exercise builds brain health: key roles of growth factor cascades and inflammation. Trends Neurosci. 30, 464-472.

de Frias, C. M., Annerbrink, K., Westberg, L., Eriksson, E., Adolfsson, R., and Nilsson, L. G. (2004). COMT gene polymorphism is associated with declarative memory in adulthood and old age. Behav. Genet. 34, 533-539.

Egan, M. F., Goldberg, T. E., Kolachana, B. S., Callicott, J. H., Mazzanti, C. M., Straub, R. E., et al. (2001). Effect of COMT Val108/158Met genotype on frontal lobe function and risk for schizophrenia. Proc. Natl. Acad. Sci. USA 98, 6917-6922. 
Egan, M. F., Kojima, M., Callicott, J. H., Goldberg, T. E., Kolachana, B. S. Bertolino, A., et al. (2003). The BDNF val66met polymorphism affects activitydependent secretion of BDNF and human memory and hippocampal function. Cell 112, 257-269.

Ettinger, U., Kumari, V., Collier, D. A., Powell, J., Luzi, S., Michel, T. M., et al. (2008). Catechol-O-methyltransferase (COMT) Val(158)Met genotype is associated with BOLD response as a function of task characteristic Neuropsychopharmacology. [Epub ahead of print]. doi: 10.1038/sj.npp.1301658

Fratiglioni, L., and Wang, H. X. (2007). Brain reserve hypothesis in dementia. J. Alzheimers Dis. 12, 11-22.

Goldberg, T. E., Egan, M. F., Gscheidle, T., Coppola, R., Weickert, T., Kolachana, B. S., et al. (2003). Executive subprocesses in working memory: relationship to catechol-O-methyltransferase Val158Met genotype and schizophrenia. Arch. Gen. Psychiatry 60, 889-896.

Hariri, A. R., Goldberg, T. E., Mattay, V. S., Kolachana, B. S., Callicott, J. H. Egan, M. F., et al. (2003). Brain-derived neurotrophic factor val66met polymorphism affects human memory-related hippocampal activity and predicts memory performance. J. Neurosci. 23, 6690-6694.

Harris, S. E., Fox, H., Wright, A. F., Hayward, C., Starr, J. M., Whalley, L. J., et al. (2006). The brain-derived neurotrophic factor Val66Met polymorphism is associated with age-related change in reasoning skills. Mol. Psychiatry 11, 505-513.

Harris, S. E., Wright, A. F., Hayward, C., Starr, J. M., Whalley, L. J., and Deary, I. J. (2005). The functional COMT polymorphism, Val $158 \mathrm{Met}$, is associated with logical memory and the personality trait intellect/imagination in a cohort of healthy 79 year olds. Neurosci. Lett. 385, 1-6.

Hayashi, M., Mistunaga, F., Ohira, K., and Shimizu, K. (2001). Changes in BDNF-immunoreactive structures in the hippocampal formation of the aged macaque monkey. Brain Res. 918, 191-196.

He, X. M., Zhang, Z. X., Zhang, J. W., Zhou, Y. T., Tang, M. N., Wu, C. B., et al. (2007). Lack of association between the BDNF gene Val66Met polymorphism and Alzheimer disease in a Chinese Han population. Neuropsychobiology 55, 151-155.

Hedden, T., and Gabrieli, J. D. (2004). Insights into the ageing mind: a view from cognitive neuroscience. Nat. Rev. Neurosci. 5, 87-96.

Ho, B. C., Andreasen, N. C., Dawson, J. D., and Wassink, T. H. (2007). Association between brain-derived neurotrophic factor Val66Met gene polymorphism and progressive brain volume changes in schizophrenia. Am. J. Psychiatry 164, 1890-1899.

Ho, B. C., Milev, P., O'Leary, D. S., Librant, A., Andreasen, N. C., and Wassink, T. H. (2006). Cognitive and magnetic resonance imaging brain morphometric correlates of brain-derived neurotrophic factor Val66Met gene polymorphism in patients with schizophrenia and healthy volunteers. Arch. Gen. Psychiatry 63, 731-740.

Ho, B. C., Wassink, T. H.. O’Leary, D. S., Sheffield, V. C., and Andreasen, N. C. (2005). Catechol-O-methyl transferase Val158Met gene polymorphism in schizophrenia: working memory, frontal lobe MRI morphology and frontal cerebral blood flow. Mol. Psychiatry 10, 287-298.

Joober, R., Gauthier, J., Lal, S., Bloom, D., Lalonde, P., Rouleau, G., et al. (2002). Catechol-O-methyltransferase Val-108/158-Met gene variants associated with performance on the Wisconsin Card Sorting Test. Arch. Gen. Psychiatry 59, 662-663.

Kimberg, D. Y., Aguirre, G. K., and D’Esposito, M. (2000). Modulation of taskrelated neural activity in task-switching: an fMRI study. Brain Res. Cogn. Brain Res. 10, 189-196.

Kramer, A. F., Hahn, S., and Gopher, D. (1999a). Task coordination and aging: explorations of executive control processes in the task-switching paradigm. Acta. Psychol. 101, 339-378.

Kramer, A. F., Hahn, S., Cohen, N. J., Banich, M. T., McAuley, E., Harrison, C. R., et al. (1999b). Ageing, fitness and neurocognitive function. Nature 400, $418-419$.

Kray, J., and Lindenberger, U. (2000). Adult age differences in task switching. Psychol. Aging 15, 126-147.

Lachman, H. M., Papolos, D. F., Saito, T., Yu, Y. M., Szumlanski, C. L., and Weinshilboum, R. M. (1996). Human catechol-O-methyltransferase pharmacogenetics: description of a functional polymorphism and its potential application to neuropsychiatric disorders. Pharmacogenetics 6, 243-250.

Liu, M. E., Hong, C. J., Liou, Y. J., Tsai, Y. L., Hsieh, C. H., and Tsai, S. J. (2008). Association study of a functional catechol-O-methyltransferase polymorphism and executive function in elderly males without dementia. Neurosci. Lett. 436, 193-195.
Lu, B. (2003). Pro-region of neurotrophins: role in synaptic modulation. Neuron 39, 735-738.

Lu, B., Pang, P. T., and Woo, N. H. (2005). The yin and yang of neurotrophin action. Nat. Rev. Neurosci. 6, 603-614.

MacDonald, A. W. III, Carter, C. S., Flory, J. D., Ferrel, R. E., and Manuck, S. B. (2007). COMT val158Met and executive control: a test of the benefit of specific deficits to translational research. J. Abnorm. Psychol. 116, 306-312.

Malhotra, A. K., Kestler, L. J., Mazzanti, C., Bates, J. A., Goldberg, T., and Goldman, D. (2002). A functional polymorphism in the COMT gene and performance on a test of prefrontal cognition. Am. J. Psychiatry 159, 652-654.

Matsushita, S., Arai, H., Matsui, T., Yuzuriha, T., Urakami, K., Masaki, T., et al. (2005). Brain-derived neurotrophic factor gene polymorphisms and Alzheimer's disease. J. Neural Transm. 112, 703-711.

Mattay, V. S., Goldberg, T. E., Fera, F., Hariri, A. R., Tessitore, A., Egan, M. F., et al. (2003). Catechol-O-methyltransferase val158-met genotype and individual variation in the brain response to amphetamine. Proc. Natl. Acad. Sci. USA 100, 6186-6191.

Mattson, M. P., Duan, W., and Gou, Z. (2003). Meal size and frequency affect neuronal plasticity and vulnerability to disease: cellular and molecular mechanisms. J. Neurochem. 84, 417-431.

Miyajima, F., Ollier, W., Mayes, A., Jackson, A., Thacker, N., Rabbitt, P., et al. (2008). Brain-derived neurotrophic factor polymorphism Val66Met influences cognitive abilities in the elderly. Genes Brain Behav. 7, 411-417.

Neves-Pereira, M., Mundo, E., Muglia, P., King, N., Macciardi, F., and Kennedy, J. L. (2002). The brain-derived neurotrophic factor gene confers susceptibility to bipolar disorder: evidence from a family-based association study. Am. J. Hum. Genet. 71, 651-655.

O'Hara, R., Miller, E., Liao, C. P., Way, N., Lin, X., and Hallmayer, J. (2006). COMT genotype, gender and cognition in community-dwelling, older adults. Neurosci. Lett. 409, 205-209.

Pang, P. T., and Lu, B. (2004). Regulation of late-phase LTP and long-term memory in normal and aging hippocampus: role of secreted proteins IPA and BDNF. Ageing Res. Rev. 3, 407-430

Pang, P. T., Teng, H. K., Zaitsev, E., Woo, N. T., Sakata, K., Zhen, S., et al. (2004). Cleavage of proBDNF by tPA/plasmin is essential for long-term hippocampal plasticity. Science 306, 487-491.

Pencea, V., Bingaman, K. D., Wiegand, S. J., and Luskin, M. B. (2001). Infusion of brain-derived neurotrophic factor into the lateral ventricle of the adult rat leads to new neurons in the parenchyma of the striatum, septum, thalamus, and hypothalamus. J. Neurosci. 21, 6706-6717.

Pezawas, L., Verchinski, B. A., Mattay, V. S., Callicott, J. H., Kolachana, B. S., Straub, R. E., et al. (2004). The brain-derived neurotrophic factor val66met polymorphism and variation in human cortical morphology. J. Neurosci. 24, 10099-10102.

Rogers, R. D., and Monsell, S. (1995). The costs of a predictable switch between simple cognitive tasks. J. Exp. Psychol. Gen. 124, 207-231.

Scharfman, H. E., and Maclusky, N. J. (2005). Similarities between action of estrogen and BDNF in the hippocampus: coincidence or clue? Trends Neurosci. $28,79-85$.

Starr, J. M., Fox, H., Harris, S. E., Deary, I. J., and Whalley, L. J. (2007). COMT genotype and cognitive ability: a longitudinal aging study. Neurosci. Lett. 421, 57-61.

Taylor, W. D., Züchner, S., McQuoid, D. R., Payne, M. E., Macfall, J. R., Steffens, D. C., et al. (2008). The brain-derived neurotrophic factor Val66Met polymorphism and cerebral white matter hyperintensities in late-life depression. Am. J. Geriatr. Psychiatry 16, 263-271.

Taylor, W. D., Züchner, S., McQuoid, D. R., Steffens, D. C., Speer, M. C., and Krishnan, K. R. (2007). Allelic differences in the brain-derived neurotrophic factor Val66Met polymorphism in late-life depression. Am. J. Geriatr. Psychiatry 15, 850-857.

Tsai, S. J., Yu, Y. W., Chen, T. J., Chen, J. Y., Liou, Y. J., Chen, M. C., et al. (2003). Association study of functional catechol-O-methyltransferase-gene polymorphism and cognitive function in healthy females. Neurosci. Lett. 338, 123-126.

van Praag, H., Kempermann, G., and Gage, F. H. (2000). Neural consequences of environmental enrichment. Nat. Rev. Neurosci. 1, 191-198.

Ventriglia, M., Bocchio Chiavetto, L., Benussi, L., Binetti, G., Zanetti, O., Riva, M. A., et al. (2002). Association between the BDNF 196 A/G polymorphism and sporadic Alzheimer's disease. Mol. Psychiatry 7, 136-137.

Winterer, G., Musso, F., Vucurevic, G., Stoeter, P, Konrad, A., Seker, B., et al. (2006). COMT genotype predicts BOLD signal and noise characteristics in prefrontal circuits. Neuroimage 32, 1722-1732. 